\section{Keeping track of the SARS-CoV-2 vaccine pipeline}

When will we have an effective vaccine against SARS-CoV-2? There can be few questions with more profound implications for what life will look like over the coming years.

On the one hand, there is a blunt and unsatisfying answer: until the results of large-scale efficacy trials are available, we simply cannot know. for optimism. This year has seen a remarkable mobilization of resources, ingenuity and dedication from vaccine researchers across the globe. More than 200 vaccine candidates are in development, and phase III efficacy trials are either underway or imminent for nine candidates spanning three different vaccine platforms.

Things are moving quickly. In response to the almost daily questions about process and progress asked by stakeholders in academia, media and politics as well as the public, the Vaccine Centre at the London School of Hygiene \& Tropical Medicine ( $\mathrm{VaC}$ ) developed an online SARS-CoV-2 vaccine development tracker in April 2020 (VaC COVID-19 vaccine tracker). On the other hand, there is cause
The VaC tracker currently contains three key features, each of which is updated on a weekly basis. The 'vaccine landscape' includes a summary of SARS-CoV-2 vaccine candidates, including platform, institutes involved and phase of development. This pools the latest information from the World Health Organization and several other online repositories. We are also very grateful for additional information provided directly by vaccine developers and welcome their continued input in the months ahead.

The 'clinical trials database' summarises key attributes of all registered SARS-CoV-2 vaccine trials, including their size, location, study design, enrolment status and estimated completion date, alongside links to clinical protocols. This resource now includes information for more than 75 registered trials and

Finally, as trials progress through the development phases, safety and immunogenicity data are becoming available on a larger scale. These need to be interpreted carefully. While reports that "vaccine new studies continue to be added.

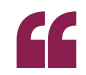

Comparing

findings

across trials

will remain a

challenge ...

but having all

the relevant

results in the

same place is a useful starting

point

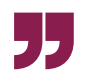

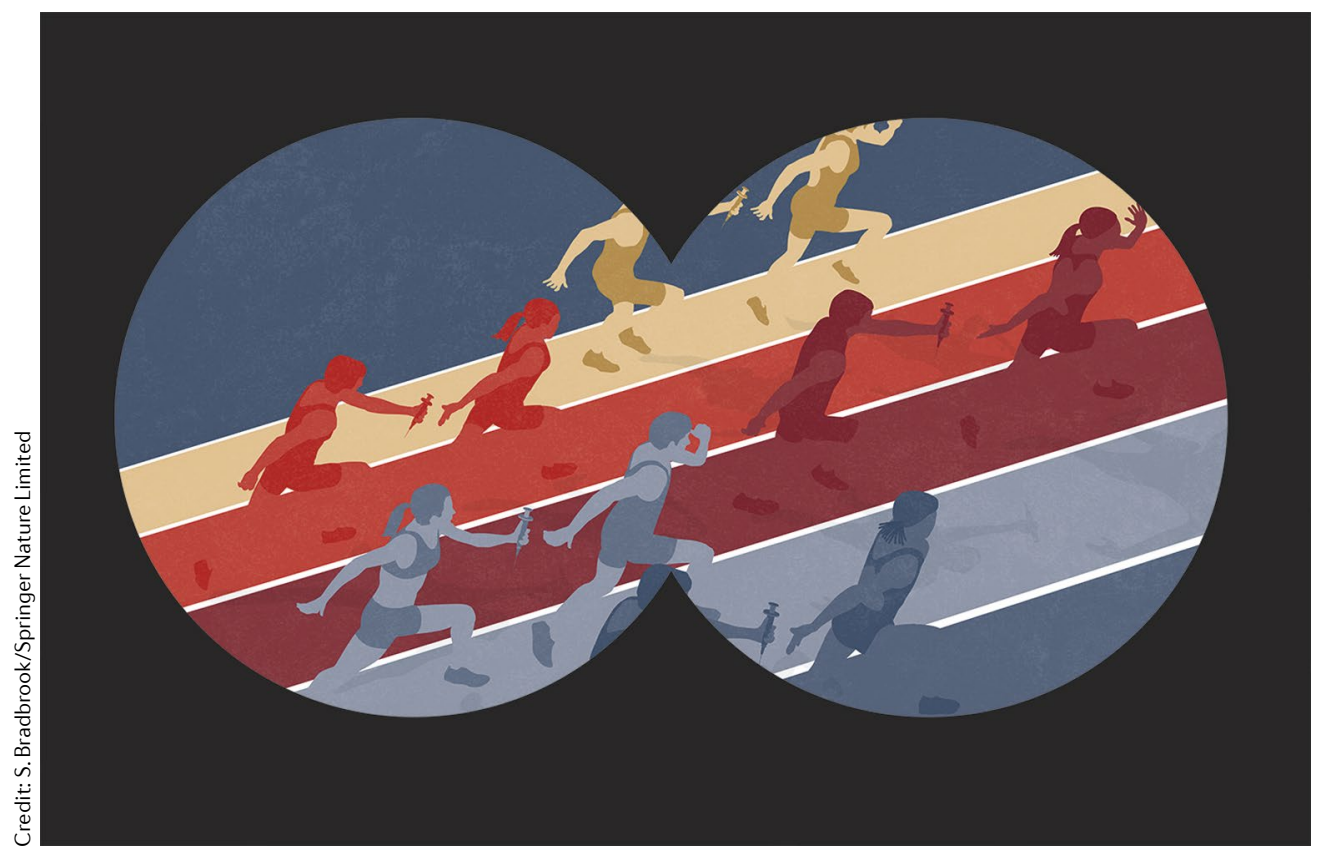

$\mathrm{X}$ induced an immune response" are common in media coverage, not all immune responses are created equal. In August 2020, we launched a 'living review' that distils the results of clinical trials as they become available. This includes a comprehensive and accessible presentation of antibody and T cell response data. Comparing findings across trials will remain a challenge owing to differences in study design, lab methods and myriad other factors, but having all the relevant results in the same place is a useful starting point.

Many challenges lie ahead. While we can hope that a safe and highly effective vaccine will emerge from the fray, we must also prepare for a situation in which we have multiple partially protective SARS-CoV-2 vaccines, conferring immunity that wanes over time. Phase III efficacy trials will tell us more, but they are not the end of the story. Stringent monitoring for safety, effectiveness and durability of protection will be essential as the number of vaccinated individuals grows.

Our tracker will adapt and expand to meet the demands of this evolving field. We plan to add new features monitoring the status and results of efficacy trials, as well as the challenges associated with distributing limited vaccine supplies in an equitable way.

The $\mathrm{VaC}$ is not directly involved in the development of any vaccines, leaving us ideally placed to provide an independent academic voice on SARS-CoV-2 vaccine development. Our motto is "Bringing vaccine research together", and we hope that our tool does just that by supplying objective and freely accessible information in the midst of the global crisis.

Edward P. K. Parker ${ }^{1 凶}$, Madhumita Shrotri ${ }^{1}$ and Beate Kampmann $n^{1,2}$

'The Vaccine Centre, London School of Hygiene \& Tropical Medicine, London, UK.

${ }^{2}$ Vaccines \& Immunity Theme, MRC Unit, The Gambia at the London School of Hygiene \& Tropical Medicine, London, UK.

凶e-mail:edward.parker@Ishtm.ac.uk

Competing interests

The authors declare no competing interests. 